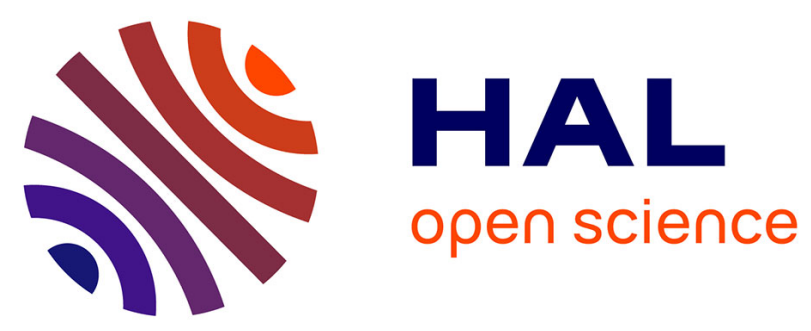

\title{
Introduction. Les indicateurs participatifs tiennent-ils leurs promesses
}

\author{
Léa Sébastien, Markku Lehtonen, Tom Bauler
}

\section{To cite this version:}

Léa Sébastien, Markku Lehtonen, Tom Bauler. Introduction. Les indicateurs participatifs tiennent-ils leurs promesses. Participations - Revue de sciences sociales sur la démocratie et la citoyenneté, 2017, 18, pp.9-38. hal-01860427

\section{HAL Id: hal-01860427 \\ https://hal-univ-tlse2.archives-ouvertes.fr/hal-01860427}

Submitted on 23 Aug 2018

HAL is a multi-disciplinary open access archive for the deposit and dissemination of scientific research documents, whether they are published or not. The documents may come from teaching and research institutions in France or abroad, or from public or private research centers.
L'archive ouverte pluridisciplinaire HAL, est destinée au dépôt et à la diffusion de documents scientifiques de niveau recherche, publiés ou non, émanant des établissements d'enseignement et de recherche français ou étrangers, des laboratoires publics ou privés. 


\section{Introduction. Les indicateurs participatifs tiennent-ils leurs promesses?}

\section{> Léa Sébastien, Markku Lehtonen, Tom Bauler}

'association des notions d'indicateurs et de participation trouve son origine au sommet de la Terre de Rio (1992), à la fois dans l'Agenda 21 qui appelle à l'élaboration d'indicateurs de développement durable au travers de prises de décision multi-acteurs, et dans la résolution 38 du rapport L'avenir que nous voulons qui réaffirme le besoin d'indicateurs alternatifs au PIB comme mesure aspirant à refléter bien-être et progrès. Émergent alors des indicateurs participatifs (économiques, sociaux et environnementaux) qui ont comme objectif de décider ensemble de ce qu'il faut mesurer (ou non) dans la société, autrement dit de démocratiser la mesure.

Un indicateur est un signal - une représentation - d'une certaine réalité qui ne se laisse pas directement observer ou expérimenter; il simplifie une couche particulière de la réalité qui par sa nature même échappe à nos sens, à nos capacités premières d'identification et de connaissance. Un indicateur let a fortiori l'entité ou la personne l'ayant configurél simplifie, réduit, et ce faisant oriente le regard de son observateur. Ainsi se multiplient les critiques des indicateurs économiques établis, et les appels pour les remplacer ou les compléter avec des données sociales et environnementales. Le PIB ne permet pas de mettre en exergue les dynamiques multidimensionnelles en œuvre au sein des territoires, et - comme l'affirment Stiglitz et al. (2010) de concert avec d'autres - «il est temps que notre système statistique mette davantage l'accent sur la mesure du bien-être de la population que sur celle de la production économique ». Ce type d'appel à complexifier ou réorienter nos mesures n'est pas nouveau en soi et rejoint ce qu'exprimait déjà Le Play (1878) au XIXe siècle : « passer de la richesse des nations au bien-être des populations ». Par contre, ce qui est beaucoup plus 
nouveau est l'appel très insistant pour penser les indicateurs et leurs reconfigurations, voire interprétations, de manière participative. La participation large des parties prenantes et des publics variés dans la conception des indicateurs est une des recommandations les plus fréquentes dans les discussions sur le travail dans ce domaine, partant du constat que les plus compétents pour savoir et décider de ce qu'est «être bien » sont les intéressés eux-mêmes. Dans cette optique, les termes de qualité de vie, de progrès social, de bien-être, de développement durable, de mieux vivre, etc. nécessiteraient d'associer ceux qui sont concernés directement par l'objet sous mesure, pour définir précisément le contenu empirique de ces expressions et ainsi ajouter discernement, légitimité et responsabilité au choix d'indicateurs pour nos sociétés (Venetoulis, Cobb, 1998).

Comme l'ont montré d'importants travaux dans le domaine de la sociologie et de l'économie politique de la mesure (Vanoli, 2002 ; Desrosières, 1993, 2008, 2015 ; Méda, Jany-Catrice, 2013), l'appareil statistique gouvernemental y compris les acteurs scientifiques qui expérimentent et préconfigurent les mesures émergentes - est outillé pour normaliser nos mesures. Néanmoins, il a de plus en plus de mal à légitimement décider des normes à utiliser dans ces exercices. Les indicateurs deviennent des objets d'interaction entre acteurs et prennent une signification politique, passant du statut d'instrument de gestion à celui d'institution de gouvernance et de démocratisation de la mesure. Dans l'idéal, les indicateurs peuvent ainsi opérer en tant qu' « objets-frontière » (Turnhout, 2009; Star, 2010) entre les «mondes sociaux » tels que science, politique et société, et ainsi satisfaire des objectifs tant analytiques que délibératifs, en combinant données statistiques, modélisation et raisonnements collectifs. Le foisonnement d'indicateurs environnementaux, de développement durable et de bien-être a été tel que certains parlent d'une «industrie d'indicateurs » (voir par exemple Hezri, Hasan, 2004). Dans ces domaines, il paraît indispensable d'associer la société civile à la production des indicateurs afin de discuter des données, variables et paramètres à prendre en compte pour s'assurer de leur cohérence avec les valeurs fondant l'objet de la mesure (comme le développement durable). Les défenseurs des indicateurs participatifs cherchent à prouver le succès et les vertus de la participation dans l'effort pour démocratiser la mesure et soulignent les critères procéduraux, parfois inspirés par les principes habermassiens d'une «situation idéale de la parole » (Van den Hove, 2006).

Cependant, peu de travaux s'intéressent à la façon dont l'information issue d'une démarche participative peut être intégrée dans le processus de construction des indicateurs, ou plus largement interrogent les apports et limites des indicateurs participatifs. Ce dossier vise à éclairer précisément ces deux points. Nous nous intéressons spécifiquement aux processus participatifs mis en place dans l'élaboration et l'utilisation de nouvelles catégories d'indicateurs socioenvironnementaux appelés Nouveaux Indicateurs de Richesse (NIR), Indicateurs de Développement Durable (IDD) et Indicateurs de Bien-Être (IBE) (voir par exemple Stiglitz et al., 2010 ; Sébastien, Bauler, 2013 ; Seaford, 2013 ; Gadrey, 
Jany-Catrice, 2012; Thiry, 2012; Jany-Catrice, 2013 ; Ruyters et al., 2011 ; IWEPS, 2015).

L'essor de ce type d'indicateurs soulève la question classique de l'articulation entre les formes de participation ascendante et descendante; entre les processus participatifs initiés d'un côté par les ONG, think tanks et associations qui proposent des indicateurs que l'on peut qualifier de contestataires, et de l'autre par les autorités et acteurs institutionnels lvoir les travaux de l'OCDE sur l'index du vivre mieux) qui développent, notamment depuis le rapport StiglitzSen-Fitoussi (2010), leurs propres démarches consultatives, participatives ou délibératives. Là où les indicateurs développés par les acteurs de la société civile ont une vertu « déstabilisatrice » en défiant les institutions existantes et en remettant en question les idées reçues et les cadres de pensée dominants, ceux issus des acteurs institutionnels, notamment des bureaux de statistiques nationaux et internationaux, ont vocation, au contraire, à standardiser et stabiliser les règles de production de la mesure. À propos de la participation, si les premiers luttent pour prolonger sa signification et multiplier son occurrence, les seconds la relaient vers des phases exploratoires liées à la configuration des indicateurs. Mais sur ce point, les bureaux de statistiques font face à un dilemme les obligeant à se positionner entre deux missions qui ont tendance à s'opposer. D'une part, nous avons l'impératif d'ouverture vers des indicateurs innovants basés sur des processus participatifs ainsi qu'une large gamme de sources d'information afin de traduire en mesures les représentations « des réalités » au sein d'une société donnée ; et d'autre part, leur mission originelle qui est de fournir des statistiques incontestables, fiables, immuables dans le temps et participant à rendre visible la réalité des gouvernants en place. Or, les IDD (Indicateurs de Développement Durable), les NIR (Nouveaux Indicateurs de Richesse) et les IBE (Indicateurs de Bien-Être) ont la vocation expresse d'engager les citoyens et usagers non seulement dans la conception des indicateurs, mais aussi dans des processus interactifs d'utilisation et d'amélioration de ceux-ci (Stiglitz et al., 2010 ; Sébastien, Bauler, 2013 ; Seaford, 2013). Depuis le début des années 1990, une multitude d'indicateurs alternatifs ont été conçus, sous l'impulsion d'une diversité d'acteurs, à des échelles variant du local au mondial. De fortes attentes sont investies dans la capacité des indicateurs alternatifs, parfois adoptés après délibération entre une large gamme d'acteurs étatiques et non étatiques, à stimuler l'action politique vers une soutenabilité accrue.

La recherche en sciences sociales qui s'est construite autour des indicateurs alternatifs montre que les indicateurs peuvent être conçus tantôt comme des outils au service de la domination et de l'oppression dans une gouvernance d'inspiration néolibérale, tantôt comme des vecteurs d'émancipation et d'apprentissage collectif qui permettent aux citoyens de mieux s'approprier les enjeux sociétaux en question et tenir les gouvernants redevables de leurs actions (Bruno et al., 2014, 2016). La vision sombre des indicateurs associés à un moyen de contrôle se nourrit notamment des expériences de l'utilisation des indicateurs de performance, par exemple dans les entreprises, tandis que 
l'espoir d'émancipation sous-tend les efforts pour développer des indicateurs locaux de développement durable ou de bien-être. Dans ce dernier cas, la participation des citoyens dans la conception d'indicateurs nourrit une multitude de promesses que nous avons identifiées et sur lesquelles nous souhaitons revenir ici : diminuer la domination des processus participatifs par les experts ; intégrer une diversité de points de vue et de perspectives; générer des apprentissages collectifs ; atténuer les asymétries de pouvoir ; favoriser l'utilisation des indicateurs par les décideurs ; permettre une meilleure intégration des considérations locales et globales.

En effet, la prolifération des expériences participatives n'est pas sans revêtir des aspects paradoxaux ou ambivalents, de même que des promesses non tenues, manipulatoires ou simplement irréalistes. La participation est-elle motivée par la volonté de réhabiliter l'action collective, ou au contraire de court-circuiter les processus démocratiques habituels institutionnalisés, et donc les forces collectives en présence? S'agit-il d'obtenir d'une population donnée l'adhésion implicite ou explicite sur des projets ou des politiques données et prédéfinies? Ou à l'inverse, cherche-t-on à véritablement organiser un partage du pouvoir de délibération et de décision plus égalitaire entre gouvernants et gouvernés ? Cherche-t-on le consensus, ou plutôt une meilleure expression des aspects sous-jacents au conflit et d'un pluralisme des vues? De telles ambiguïtés, limites, tensions et dilemmes - depuis longtemps au cœur d'une littérature scientifique sur la participation - semblent jusqu'ici n'avoir fait que peu d'écho dans la littérature concernant les indicateurs. Cela alors même que les appels pour des indicateurs participatifs sont légion depuis un certain temps, et que les expérimentations et opérationnalisations se multiplient. De toute évidence, les indicateurs ont leurs spécificités - ne serait-ce que parce qu'ils naviguent entre interprétation et savoir, entre utilisation instrumentale de la participation et processus de démocratisation citoyenne.

Ce dossier présente au lecteur une sélection de cinq contributions originales d'analystes des indicateurs participatifs. Elles s'organisent toutes autour d'une série de promesses des indicateurs participatifs, sources de paradoxes et dilemmes. Ces contributions abordent différentes facettes de ces promesses, et les multiples couches analytiques permettent d'approfondir l'étude de processus d'indicateurs participatifs qui furent opérationnalisés, tout comme des réflexions théoriques et conceptuelles à la confluence des théories de la participation et de la sociologie de l'information. Ce dossier vise à consolider et rassembler des réflexions qui sont actuellement menées de manière éparse au sein de l'espace de recherche francophone sur l'intersection entre participation et indicateurs. Jusqu'à présent - et nos contributions le montrent à un certain égard -, cette réflexion est surtout menée depuis le champ de l'expertise en indicateurs. Le dossier se veut ainsi être une main tendue vers le champ de l'analyse critique des processus et impacts participatifs, afin de pouvoir avancer ensemble sur un programme de travail commun. 


\section{Questionnement central : les six promesses des indicateurs participatifs}

On trouve un consensus dans la littérature sur la nécessité de développer des indicateurs participatifs : il s'agit d'un appel à une collaboration plus étroite entre les producteurs et les potentiels utilisateurs des indicateurs, afin que ceux-ci répondent aux «vraies » préoccupations sociétales (Batterbury, Forsyth, 1997 ; Nygren, 1999 ; Turcu, 2013 ; POINT, 2011 ; Bell, Morse, 2003 ; Rondinella et al., 2017). L'état de l'art qui suit nous amène à identifier dans la littérature six grandes vertus de la participation élargie des parties prenantes dans la proposition d'indicateurs alternatifs, six vertus que le dossier vise à illustrer et contextualiser. Ces vertus sont stylisées sous forme de promesses que se font les acteurs des indicateurs participatifs en misant sur la refonte de nos mesures communes. Avant tout, changer de mesure en changeant le processus de son élaboration est supposé amener à changer la société - une promesse ultime que poursuivent toutes les expériences participatives d'indicateurs alternatifs.

\section{Promesse 1 : empowerment des citoyens, transformations des rôles de l'expert}

En premier lieu, l'idée d'associer participation et indicateurs vise à émanciper les citoyens au niveau individuel afin que ceux-ci gagnent les compétences nécessaires pour s'insérer dans les transformations sociétales visées. Il s'agit de contrebalancer et de modifier le rôle dominant de l'expert dans la conceptualisation des indicateurs (Innes, Booher, 2000 ; Bell, Morse, 2003 ; Rydin et al., 2003 ; Hezri, 2006 ; Reed et al., 2006 ; Holden, 2013 ; Turcu, 2013). La notion d'empowerment est ici beaucoup utilisée pour insister sur l'effet émancipatoire des processus participatifs autour de choix de mesure pour la société (Salvaris, 2000). La multiplication des expériences de production des NIR, IDD ou IBE repose en grande partie sur un scepticisme croissant à l'égard de l'expertise institutionnalisée incarnée dans les structures de prise de décision. Or, les indicateurs participatifs en tant que vecteurs d'émancipation citoyenne sont fortement remis en cause par l'ensemble des contributions de ce dossier; le rôle des experts semble rester prédominant dans les processus étudiés.

Deux modalités de mise en relation entre les experts et les citoyens peuvent être observées : proposer une discussion cadrée basée sur une liste préformatée d'indicateurs, ou proposer une discussion libre sur les valeurs et indicateurs sans aucune donnée préalable.

Le premier cas-discussion cadrée par l'expertise conventionnelle-apparaît comme le plus courant dans la littérature comme dans les contributions de ce dossier. Étant donné la nature éminemment technique des indicateurs - institutionnalisée dans les batteries d'indicateurs officiels, opérations et procédures de normalisation et bureaux de statistiques nationaux -, la tendance des cadrages 
définis par les experts apparaît particulièrement prégnante dans les processus participatifs dans ce domaine (Bogner, 2012). Dans ce dossier, Florence JanyCatrice et Samuel Pinaud présentent trois cas très différents de participation autour d'indicateurs : les indicateurs de bien-être du CESE, l'Indicateur de Santé Sociale de la Région Nord-Pas-de-Calais et un sondage sur l'habitat participatif. Malgré les méthodologies très éclectiques pour organiser la participation, cette dernière est toujours centrée sur les experts, la discussion étant fortement cadrée par la liste des indicateurs proposés en amont du processus de participation. Ce préformatage ne permet aucun débat plus profond, notamment sur les choix de configuration ou de conventionnement des mesures développées, mais valide un projet élaboré ex ante pour lequel un supplément de légitimité était attendu. Les experts justifient le fait de proposer une première liste d'indicateurs aux participants par l'objectif d'avoir une base de discussion et éviter l'éparpillement, mais une telle procédure oriente la discussion vers une issue attendue.

Le second cas - discussion libre, sans cadrage strict - semble beaucoup moins courant car plus difficile à organiser et à restituer. Même quand une telle approche est adoptée, l'expertise quantitative tend à cadrer indirectement le processus. Comme le montrent les cas étudiés par Michel Renault, le dispositif peut jouer un rôle de référent silencieux (Tournay, 2015) ou, selon les mots de Marie-Hélène Vergote et Sandrine Petit (dans ce dossier), de «technologie invisible » selon laquelle les éléments de structuration du réel engendrent des choix et comportements qui échappent à la prise des hommes. Qui plus est, les données proposées par les groupes de citoyens à la suite de processus participatifs ouverts peinent à être prises au sérieux par les experts et décideurs, notamment en raison de préoccupations concernant leur crédibilité, comparabilité et complétude (Gouveia et al., 2004 ; Bradshaw, 2003 ; Conrad, Hilchey, 2011). Les experts développant des indicateurs sont, par conséquent, souvent frileux à l'idée d'une participation élargie à la conception des indicateurs, évoquant les risques de «politisation » d'une matière qu'ils jugent devoir être objectivante et exempte de toute réalité partisane. Étant donné leur caractère quantitatif et statistique, les indicateurs donnent l'impression d'être de parfaites opérationnalisations d'une rationalité «technoscientifique», marginalisant ainsi les autres formes de connaissances et de rationalités. Les débats autour des indicateurs sont le plus souvent organisés autour d'aspects techniques (Bora, Hausendorf, 2006), ce qui conduit à une dépolitisation des enjeux associés à la mesure.

Dans les deux cas, ces nouveaux indicateurs participent à des processus d' « instrumentation » (Lascoumes, Le Galès, 2005) par quantification, renforçant et institutionnalisant ainsi le pouvoir de l'expert. De même, les indicateurs rendent les enjeux accessibles aux profanes, mais ces procédures de simplification, en elles-mêmes, légitiment le rôle de l'expert comme le seul capable de «maîtriser le chiffre » (Zittoun, 2006). L'ensemble des contributions de ce numéro soulignent le rôle privilégié des économistes, des ingénieurs et des bureaux 
d'étude dans la conception des indicateurs participatifs. Malgré la réticence, certes compréhensible, des experts à mettre en danger leur autorité et à s'attendre à l'inattendu, Anne Le Roy et Fiona Ottaviani (dans ce dossier) évoquent la capacité des expérimentations portées par le monde associatif à bousculer les paradigmes institutionnalisés.

\section{Promesse 2 : intégration des subjectivités, des discours, des diversités de points de vue}

La seconde promesse des indicateurs participatifs concerne la prise en compte des subjectivités, des représentations individuelles, de la diversité des points de vue et des discours des différents acteurs dans la construction de la mesure (Innes, Booher, 2000, p. 173 ; Bell, Morse, 2001 ; Rydin et al., 2003 ; Hezri, 2006, p. 114 ; Rafols et al., 2012 ; Turcu, 2013). En particulier, la participation servirait à construire un système d'intelligibilité capable d'intégrer aussi les dimensions subjectives du bien-être et du développement durable (Citton, 2007 ; Stiglitz et al., 2010).

On observe que les processus d'indicateurs participatifs oscillent entre deux extrêmes concernant l'objectif premier de la participation : (1) faire émerger la diversité des points de vue via un débat ouvert, par exemple sur la signification de la vie bonne, ou (2) permettre la consolidation d'un consensus via un débat resserré, par exemple sur un indicateur de développement durable à l'échelle locale. Dans le premier cas, la participation sert à libérer la parole à la fois sur les indicateurs et sur les valeurs qu'ils véhiculent afin de générer une discussion riche. Toutefois, les résultats de ces échanges seront difficilement restituables sans perte d'information, et la discussion pourra souffrir d'un manque de structure et d'apport scientifique. Dans le second cas, les porteurs des projets visent souvent la mise en place de débats relativement techniques qui portent, par exemple, sur la qualité des données, les paramètres, les agrégations, les effets spatiaux.

Ce dernier cas de figure est le plus fréquent, notamment car il est plus facile à mettre en œuvre. Le processus suit alors des étapes bien balisées de la configuration d'une mesure; elle est peut-être aussi plus facile à défendre face à des bailleurs de fonds et acteurs politiques qui sont en attente de résultats et produits tangibles. Sa tendance à favoriser la recherche d'un consensus limite évidemment un des apports principaux de la participation, c'est-à-dire le traitement ouvert des controverses. Selon les termes de Renault (dans ce dossier), évacuer les « contes » au profit des «comptes » est une erreur, notamment sur des thématiques fondamentales comme le bien-être ou la qualité de vie. Sur ces sujets qui en deviennent vite des projets de société, avant de s'occuper de la donnée, il faudrait appréhender les relations entre participants, les débats sur les valeurs de la société et explorer l'articulation entre paroles singulières et mesure. 
Comme le dit Renault dans son article, «l'engagement dans une "enquête sociale" apparaît largement impensé dans le passage à des indicateurs ». Or, ce sont souvent les à-côtés qu'amène une réflexion sur les indicateurs qui s'avèrent les plus intéressants et permettent de mieux rendre compte des situations vécues et expérimentées par les participants. Mais même lorsque le processus participatif est méthodologiquement bâti sur les échanges ou la parole libre, le traitement des données recueillies n'échappe pas à l'agrégation des préférences (souvent au travers d'une lexicométrie basée sur le nombre d'occurrences), et ne rend donc pas compte du pluralisme des interprétations et de l'incommensurabilité des valeurs.

Les contributions de Jany-Catrice et Pinaud, Le Roy et Ottaviani, et Vergote et Petit mettent le doigt sur cette tension permanente entre la fermeture des débats liée à la recherche de consensus et l'ouverture des perspectives en vue de rendre productifs les conflits. En effet, la notion de conflits et controverses productifs va à l'encontre de deux hypothèses qui sous-tendent souvent le travail de production et d'utilisation des indicateurs (Parris, Kates, 2003 ; Rauschmayer, Wittmer, 2006). D'abord, pour être influents dans la prise de décision, les indicateurs doivent faire l'objet de consensus concernant leur forme et les valeurs et hypothèses épistémiques sous-jacentes (Godin, 2002 ; McCool, Stankey, 2004) ; ensuite, les indicateurs visent à générer des consensus sur des questions politiques (tant sur les objectifs que sur les moyens).

Selon Mitroff et Emshoff (1979, p. 10), le risque n'est pas le consensus lui-même, mais un consensus précoce, conclu pour de mauvaises raisons, par exemple l'incapacité de tolérer le conflit. Ces risques sont particulièrement aigus lorsque les différentes parties prenantes impliquées s'attachent à des systèmes de valeurs très contrastés (Hisschemöller, Cuppen, 2015, p. 42). Par ailleurs, un processus orienté vers le consensus risque de reléguer au second plan la qualité de l'information sous-jacente, comme la robustesse d'un indicateur (Rydin, 2007a, p. 55-56; Petersen et al., 2011).

Les vertus potentielles associées aux controverses et aux conflits ont été soulignées par exemple par les théories de la démocratie agonistique (Mouffe, 1999) et la sociologie pragmatique (Chateauraynaud, 2011 ; Chateauraynaud, Debaz, 2017). Dans cette perspective, les indicateurs pourraient éclairer les fondements sous-jacents aux conflits existants, favoriser l'apprentissage en remettant en question les cadres de pensée dominants et les idées reçues, ainsi qu'ouvrir des perspectives (voir par exemple Stirling, 2008). Suivant le constat de Barthe (2002), le succès d'une expérimentation participative ne s'évalue pas à l'aune de sa capacité à régler des conflits, mais plutôt par le degré auquel elle permet de rendre productifs ces conflits en les transformant. Söderman et al. (2012) affirment que, dans l'idéal, les débats concernant les indicateurs concrets rendent accessible le processus politique à des parties prenantes ; les indicateurs seraient alors utilisés comme outils de planification pour favoriser la discussion et la prise de décision et non pas comme «la vérité absolue », 
source de réponses immédiates. Les contributions de ce dossier font état des capacités des divers processus d'élaboration et d'utilisation des indicateurs participatifs à résister à la tentation d'un consensus prématuré, sans toutefois trouver de voie satisfaisante pour parvenir à la prise en compte d'opinions divergentes.

\section{Promesse 3 : apprentissages croisés, élargissement des perspectives}

La discussion sur le dilemme entre consensus et diversité des points de vue nous conduit à la troisième promesse des indicateurs participatifs, à savoir la possibilité d'apprentissages croisés et d'élargissement des perspectives à travers les processus collaboratifs de conception des indicateurs (Mickwitz, Melanen, 2009 ; Lehtonen, 2013). En ouvrant les processus d'élaboration des indicateurs à de multiples groupes et types de connaissances - dont l'expertise « savante » et «profane »-, la participation engendrerait des apprentissages au sein des différents groupes impliqués, ainsi que de manière croisée. Cette juxtaposition des perspectives et des cadrages sous-jacents divers contribuerait à l'émergence d'une «gouvernance réflexive » (Voss, Bauknecht, Kemp, 2006). Comme le dit Martin (2016), il s'agit là d'« incarner une pensée critique pour ouvrir le champ des possibles ».

La littérature sur le sujet fait état de différents types d'apprentissages attendus de la participation. D'abord, l'apprentissage en simple boucle (Argyris, Schön, 1978) ou apprentissage instrumental (Hezri, Dovers, 2006) permettrait aux participants d'apprendre sur les thèmes et enjeux spécifiquement visés par les indicateurs (les données concernant la qualité de l'environnement, les inégalités sociales, etc.). Ensuite, à travers la confrontation des valeurs, visions et savoirs divers, la participation peut engendrer des apprentissages collectifs plus complexes, « en double boucle » ou en «triple boucle», portant respectivement sur les hypothèses sous-jacentes aux actions politiques ou sur les normes et cadres de pensée fondamentaux (Argyris, Schön, 1978 ; Reed et al., 2006). Cet apprentissage en lui-même peut revêtir une dimension politique ou stratégique : les participants apprennent alors à utiliser les indicateurs et leurs processus d'élaboration pour faire avancer leurs propres objectifs, légitimer leurs propres positions et actions et délégitimer celles de leurs adversaires; bref, ils apprennent à influencer les agendas politiques (Ortega-Cerdá, 2005 ; Hezri, Dovers, 2006). En d'autres mots, on observe que les acteurs développent des stratégies qui découlent de connaissances fines sur les rouages de la gouvernance contemporaine; les processus participatifs autour des indicateurs deviennent alors des véhicules pour que participants et organisateurs développent des formes de «knowing governance » (Voss, Freemann, 2016).

Les cas d'étude du dossier montrent des résultats nuancés tant au niveau des apprentissages générés que pour l'ouverture des perspectives auprès des participants. En général, les parties prenantes des arènes participatives retirent 
des informations à partir des échanges entre acteurs, autant sur les indicateurs que sur leurs perceptions sous-jacentes. L'objectif d'apprentissage représente même un leitmotiv poussant les acteurs à accepter de participer à ces discussions souvent chronophages. C'est notamment ce que soulignent Jany-Catrice et Pinaud dans leur étude sur les circuits courts, où les participants ont accepté de participer aux débats «dans un souci de sociabilité » et pour « développer leur capacité d'expertise », cherchant donc des apprentissages en simple boucle. Mais les auteurs de ce dossier constatent un décalage important entre les attentes initiales des participants et leurs réels apprentissages. Et surtout, les apprentissages que génèrent les indicateurs semblent rarement croisés, mais organisés par les experts en direction du public. Comme l'affirme Boulanger ldans ce dossier), le système politique fabrique au travers de participations citoyennes les savoirs dont il estime avoir besoin ; aussi les transferts de connaissances apparaissent-ils très cadrés et unidirectionnels.

En ce qui concerne la capacité des processus participatifs à renouveler les perspectives des acteurs, les résultats sont là encore ambivalents. D'un côté, dans le cas étudié par Vergote et Petit, malgré la finalité affichée de choisir un indicateur précis, les discussions entre acteurs ont traité de préoccupations beaucoup plus étendues, et l'espace de la participation sur les indicateurs s'est élargi sur les valeurs et expériences, générant des apprentissages en double ou triple boucle. Néanmoins, même si ces discussions sont en général identifiées comme riches en soi, on retrouve ici la difficulté précédemment évoquée : comment rendre compte de ces débats, surtout lorsque l'objectif initial fixé par l'organisateur est la recherche d'un consensus autour d'une mesure ? L'élargissement et la confrontation des perspectives sont en quelque sorte non prévus dans le déroulement de la participation et arrivent alors comme une bonne surprise pour les participants, surtout lorsque les organisateurs parviennent à rendre compte de ces débats sur les valeurs - une moins bonne surprise pour d'autres, qui ne savent pas gérer ce « débordement » et tentent toujours de ramener le débat à un choix technique d'indicateurs. On retrouve la tension entre les objectifs d'ouverture - d'apprentissage en double ou triple boucle - et le besoin de structurer le débat, voire de le fermer afin d'arriver à un accord.

\section{Promesse 4 : rééquilibrage des pouvoirs, enrichissement de la démocratie}

La quatrième promesse des indicateurs participatifs prévoit un enrichissement de la démocratie grâce à une diminution de l'asymétrie des pouvoirs (Hall, Rickard, 2013 ; Salvaris, 2000). Une large participation serait un gage de légitimité (Hardi, Zdan, 1997) qui favoriserait l'implication active des citoyens, dans l'esprit d'une démocratie délibérative. Elle diminuerait aussi le risque d'utilisation stratégique des indicateurs, par exemple pour cacher certaines évolutions sociétales, justifier des décisions déjà prises, ou retarder la prise de décision (Hezri, 2004 ; Magee et al., 2013). Un tel renouveau démocratique 
est - par exemple - évoqué à propos des sujets de développement durable et de bien-être, que la société civile prendrait à bras-le-corps pour proposer de nouvelles voies à suivre pour les sociétés via de nouveaux indicateurs. Néanmoins, les indicateurs alternatifs présentent un paradoxe entre le contenu même de toute mesure alternative et les impacts attendus de l'opérationnalisation de la mesure. La raison d'être des mesures alternatives repose sur leur capacité à remettre en question les conventions existantes des mesures du progrès et du bien-être (notamment le PIB), tandis que de par leur nature même, les indicateurs participent au renforcement de l'agenda politique managérial qui repose sur des dynamiques de quantification touchant tous les domaines de la société (Rottenburg, Merry, 2015, p. 10). Ces mêmes indicateurs alternatifs - par leur mécanique performative - sont donc employés afin d'établir des mécanismes de contrôle démocratique concernant la prise de décision politique.

Ainsi la participation autour d'indicateurs peut-elle occasionner des effets inattendus, voire aller à l'encontre des objectifs démocratiques plus larges dans lesquels s'inscrivent les indicateurs alternatifs. Dans ce dossier, Boulanger revisite ces paradoxes lourds par la perspective de la sociologie luhmannienne. Luhmann montrait que la participation peut renforcer l'effectivité des décisions, mais ne satisfait pas d'idéal démocratique, puisque l'exercice participatif débouche quasi nécessairement sur un consensus qui se forme au sein de la sphère de discussion prévue par son organisateur. Pourtant, nous dit Boulanger, la participation devrait pouvoir offrir la possibilité d'un changement de cap radical avec des conséquences imprévisibles pour la définition de la mesure et pour les processus politiques. Dans la réalité, les multiples expérimentations nous montrent que, de par les effets de cadrage forts, les indicateurs participatifs, même alternatifs, ramènent inlassablement vers les conventions en place, ce que Boulanger associe tantôt à une régression, tantôt à une imposture.

Cette thèse rejoint l'analyse de Vergote et Petit qui déplorent la validation de propositions préétablies et les asymétries de pouvoir. En parallèle, leur article illustre les moyens qu'ont les acteurs de terrain de s'émanciper en échappant aux effets potentiellement néfastes de la «mise en chiffre », à travers des processus de «mise en mots». Ces formes de contournement des indicateurs potentiellement oppressants rejoignent celles évoquées par Bruno et al. (2014) qui font état des approches ascendantes de participation dans la construction et l'utilisation des indicateurs à des fins de contestation de l'hégémonie de la quantification. L'ensemble des expérimentations d'indicateurs participatifs abordées dans ce dossier soulignent l'importance d'étudier l'articulation des dispositifs participatifs avec un ensemble d'institutions, de pratiques et de jeux d'acteurs - que l'on appelle cela un « système délibératif » (Mansbridge, 1999), une «délibération macro » (Parkinson, 2006), ou encore une «sphère discursive » (Hendriks, 2006).

De même que les indicateurs ont été conceptualisés comme un outil de «gouvernementalité » (voir par exemple Russell, Thomson, 2009) et de contrôle 
à distance, dans l'esprit de la «gouvernance par le chiffre » (voir notamment Lascoumes, Le Galès, 2005 ; Hood, 2007 ; Rydin, 2007b ; Jackson, 2011), la participation a été perçue et parfois fortement critiquée (voir par exemple Cleaver, 1999 ; Cooke, Kothari, 2001) de la même perspective. Levidow (2007) affirme que des formes de participation «domestiquée » représentent un outil de gouvernance (notamment néolibérale) destiné à éviter les conflits à travers une gestion des conflits en amont, ce qui conduit à dépolitiser le débat. Ainsi mis ensemble, ces deux outils - participation et indicateurs - se voulant être porteurs d'une certaine alternativité (de la mesure et de la manière de faire société) courent le risque de favoriser l'utilisation instrumentale et stratégique par les acteurs dominants et de ne pas parvenir à réduire les asymétries de pouvoir.

\section{Promesse 5 : augmentation de l'utilisation des indicateurs dans la prise de décision}

La cinquième promesse, peut-être la plus répandue, voit la participation comme un moyen de favoriser la légitimité et donc l'utilisation des nouveaux indicateurs ainsi produits (Innes, Booher, 2000, p. 173 ; Bell, Morse, 2003, 2008 ; Rydin et al., 2003 ; Hezri, 2006, p. 114 ; Mascarenhas et al., 2014). Les indicateurs sont, en effet, rarement utilisés de manière directe et instrumentale dans les prises de décision politiques (Sébastien, Bauler, 2013 ; Sébastien et al., 2014 ; Lehtonen, 2013 ; Lyytimäki et al., 2013). Ainsi, la fonction d'interface des indicateurs (entre politique, science et sociétél a rarement l'occasion d'opérer.

Renforcer la collaboration entre les producteurs et les potentiels utilisateurs des indicateurs pourrait en partie pallier ce problème, car elle induit une connaissance plus fine - et donc a priori une meilleure prise en compte des attentes des utilisateurs. Intégrer systématiquement de la participation à la construction des NIR, IDD ou IBE contribuerait à leur utilisation par les décideurs politiques, les approches participatives de développement des indicateurs s'appuyant sur l'hypothèse d'une chaîne de cause à effet linéaire : plus de participation $\rightarrow$ plus de démocratie $\rightarrow$ moins de dominance des experts $\rightarrow$ davantage d'utilisation des indicateurs $\rightarrow$ meilleures décisions politiques. Néanmoins, l'expérience permet de remettre en cause cette hypothèse d'un cercle vertueux automatique. S'inscrivant dans des contextes plus larges, les processus participatifs peuvent favoriser ou compromettre les efforts pour renforcer l'utilisation des indicateurs alternatifs et la probabilité que cette utilisation produise des impacts désirables pour la société.

La plupart des auteurs de ce dossier déplorent le manque d'utilisation directe des indicateurs proposés dans le cadre d'arènes participatives. À partir de l'étude de cas ISBET en Bretagne, Renault montre que les résultats de l'exercice participatif sont rarement utilisés par les décideurs. Comme nous expliquent Jany-Catrice et Pinaud, certains acteurs choisissent de quitter le processus participatif et remettre en cause la participation elle-même, pour dénoncer ce manque de 
débouchés directs. Boulanger met en exergue que les indicateurs participatifs - en se positionnant contre les institutions dominantes en place - remettent en question l'autonomie du système scientifique tout comme celle du système politique. Par contre, face au cadre politique actuel, les mécanismes participatifs ne peuvent pas réellement susciter de renouveau démocratique ni même un usage renforcé des indicateurs alternatifs élaborés. Cette quasi-opposition entre scientifiques et politiques revient souvent dans le texte de Boulanger qui souligne que la participation autour des indicateurs est tiraillée entre ces deux pôles, organisée par les scientifiques mais pour des arguments politiques. Au final, n'étant pas le fruit de demandes politiques directes, les indicateurs participatifs sont rarement appliqués dans les sphères décisionnelles.

En effet, les processus d'élaboration des indicateurs participatifs, souvent encadrés par des scientifiques, font face au même défi que la constitution des «mini-publics», un dispositif de démocratie délibérative/participative désormais répandu. L'élaboration des indicateurs participatifs a rarement lieu in situ, c'est-à-dire sur le lieu où doivent se prendre les décisions, mais plutôt ex situ, «en laboratoire», c'est-à-dire en dehors des sphères décisionnelles. Deux considérations en découlent. D’abord, le cadre participatif ex situ négligerait les réalités politiques dans lesquelles se déroulent ces expériences (Bogner, 2012), occultant ainsi les liens souvent ténus entre la participation et la prise de décision (Mazeaud, 2012), et compromettrait l'objectif d'apprentissage collectif. Ensuite, selon Hisschemöller et Cuppen (2015), les démarches participatives ex situ bénéficient d'un environnement d’apprentissage protégé, où les participants se sentent à l'aise pour s'exprimer et agir en sécurité, mais fonctionnent toutefois en vase clos et sont alors dépourvues d'un conflit authentique, sans lequel l'apprentissage social a du mal à prospérer. On en revient alors à la première impasse que nous avions identifiée, à savoir choisir entre une participation cadrée selon les perspectives et intérêts des groupes dominants, ou libre, génératrice d'apprentissages, mais non utilisée dans la prise de décision. Et lorsque c'est la sphère politique qui organise le processus, le risque est de régresser en «policy-based evidence », à savoir l'utilisation des processus d'indicateurs participatifs pour légitimer les décisions déjà prises et renforcer les cadrages dominants.

On peut certes regretter la faible utilisation directe des indicateurs participatifs dans la prise de décision, mais l'expérience passée nous invite à traiter avec prudence l'objectif même d'une utilisation de la donnée dans la sphère politique. L'utilisation d'un indicateur n'augmente pas forcément son impact et cet impact n'est pas nécessairement en phase avec les objectifs initiaux de la mesure alternative, ou encore avec les objectifs de la société dans son ensemble (voir par exemple Lyytimäki et al., 2013 ; Lehtonen et al., 2016). Par conséquent, il conviendrait de mieux conceptualiser et préciser les concepts d'utilisation et d'impact. Boulanger (dans ce dossier) souligne cette dualité à travers la grille de lecture luhmannienne: l'utilisation relèverait ainsi de «l'adaptation des structures et programmes d'un système suite à ses propres observations de 
l'environnement », tandis que l'influence consisterait dans les réactions d'un système à « la façon dont il est lui-même observé par les autres ». Jany-Catrice et Pinaud (dans ce dossier) soulignent qu'il convient de se demander à qui et à quoi profitent les indicateurs ; il ne suffit pas de chercher à maximiser l'utilisation d'un indicateur, car pour que celle-ci produise des effets positifs, l'indicateur et son utilisation doivent se conformer aux principes démocratiques acceptés par la communauté en question. L'utilisation peut être jugée - selon le cadre normatif adopté - comme abus, légitimation, manipulation stratégique, utilisation symbolique, ou simplement comme «mauvaise » utilisation provoquée par le manque de connaissances des utilisateurs. Finalement, insister sur l'utilisation des indicateurs revient à faire abstraction non seulement des impacts (comme l'apprentissage collectif) provenant des processus de leur construction, mais aussi des impacts systémiques générés par l'ensemble des dispositifs et contextes politiques dans lesquels se développent et s'emploient les indicateurs. Les modes de gouvernance spécifiques, les perspectives, les points de vue et les valeurs incorporés dans les systèmes d'indicateurs occasionnent des impacts inattendus qu'une attention exclusive sur l'utilisation ne saurait capturer (voir par exemple Lascoumes, Le Galès, 2005 ; Zittoun, 2006 ; Jackson, 2011).

\section{Promesse 6 : imbrication des enjeux multi-scalaires}

La dernière promesse que nous souhaitons interroger est celle consistant à relier les enjeux de différentes échelles, du local au global. La participation à la définition de la mesure permettrait de mieux territorialiser les notions de développement durable et de bien-être en intégrant les préoccupations locales (selon le niveau géographique concerné) dans la conception des indicateurs. Les acteurs territoriaux pourraient alors définir les aspects de soutenabilité qu'ils jugent pertinents, selon leurs propres priorités et systèmes de valeurs (Pintér et al., 2005 ; Reed et al., 2006). L'implication des parties prenantes contribuerait à mieux arrimer les objets en discussion, c'est-à-dire les mesures alternatives, aux contextes et réalités des acteurs.

Il y a une quinzaine d'années déjà, Theys (2002) évoquait le risque d'autonomisation des démarches locales par rapport aux cadres globaux, entre les acteurs du territoire et les acteurs internationaux. Là où les premiers cherchent principalement à intégrer les préoccupations environnementales dans le développement local et l'aménagement du territoire, les acteurs internationaux léconomistes, grandes entreprises, ONG internationales) s'appuient sur certains grands principes (tels que la précaution ou la consommation éthique), développent des instruments économiques ou s'engagent dans des négociations internationales. C'est dans cette tension permanente entre le local/territorial et le global/international que se situent les initiatives de production et d'utilisation des indicateurs participatifs (Fraser et al., 2006). Les indicateurs «descendants », développés par les organismes internationaux ou d'autorités à l'échelle nationale, souffrent souvent d'une faible pertinence au niveau local, tandis que les indicateurs 
«ascendants», développés à travers des processus participatifs à l'échelle locale, ne parviennent pas à s'institutionnaliser et ont du mal à se positionner dans le contexte global du développement durable ou du bien-être.

On peut constater que si les indicateurs classiques type PIB ou taux de chômage sont généralement mesurés au niveau national et définis au niveau supranational Inotamment pour permettre les comparaisons entre nations qui requièrent une très forte standardisation internationale de la définition de la mesure), la plupart des indicateurs alternatifs sont proposés au niveau infranational (local, départemental, régional). Le thème de l'interaction entre le local et le régional est peu développé dans la littérature comme dans la pratique, avec l'exception notable du Royaume-Uni, dont les indicateurs de développement durable ont été conçus afin de permettre leur application à des échelles variées (Mascarenhas et al., 2014). L'ensemble des contributions de ce dossier analysent d'ailleurs des processus au niveau infranational : l'ISBET en Bretagne, les NIR en Pays de Loire, l'ISS en Pas-de-Calais, la qualité de vie à Jacksonville, le QUARS dans une région en Italie, l'IWEPS en Wallonie ou l'IBEST à Grenoble. Seul le cas du CESE (JanyCatrice et Pinaud, dans ce numérol est abordé ici en tant qu'initiative nationale, mêlant consultations en ligne, questionnaires et panels citoyens. Ces expériences montrent que l'Europe et la France ont été pionnières dans la réflexion autour des indicateurs participatifs, notamment au niveau régional (Méda, 1999 ; Gadrey, Jany-Catrice, 2013 ; Jany-Catrice, 2007, 2009). Les travaux de la commission Stiglitz-Sen-Fitoussi (Stiglitz et al., 2010) ont donné à ce travail français une visibilité internationale. Néanmoins, les contributions dans ce dossier confirment le constat que font Chancel et al. (2014) : ces expériences, bien que de plus en plus nombreuses à l'échelle territoriale, conservent un caractère ponctuel et isolé, et leurs organisateurs peinent à en faire remonter les résultats et à rendre effectif l'utilisation des nouveaux indicateurs proposés à l'échelle nationale.

Pour répondre à la demande de comparabilité et pour remédier aux problèmes d'interaction entre échelles, Valentin et Spangenberg (2000) préconisent des indicateurs participatifs spécifiques pour chaque communauté mais inscrits dans un cadre commun, tandis que Mascarenhas et al. (2014) testent un cadre commun pour l'élaboration des indicateurs pour les municipalités d'une même région. Néanmoins, pour les indicateurs alternatifs, la demande de comparabilité entre les entités territoriales - l'utilisation d'indicateurs communs sur des territoires différents - relève d'un paradoxe : changer la mesure statistique afin de s'émanciper d'une comptabilité qui uniformise l'organisation et la représentation de la société apparaît comme le principal objectif de la volonté d'aller «au-delà du $\mathrm{PIB} »$. Pourtant, proposer une mesure alternative commune à toutes les entités territoriales reviendrait à ignorer leurs particularités, et menacerait à nouveau de dépendre d'une organisation sociétale détachée de leurs besoins spécifiques. Se pose donc la question de la souveraineté de ces territoires qui, en mettant en place un indicateur standardisé à l'échelle nationale ou internationale, sont contraints de se comparer à des entités qui ne sont pas nécessairement comparables et risquent ainsi de perdre l'opportunité de maîtriser leur avenir en 
fonction de leurs ressources propres (Thiry et al., 2016). Un exemple observé au Pays de Galles montre deux counties ${ }^{1}$ ayant appliqué à leur échelle la méthodologie du Happy Planet Index du think tank New Economics Foundation (NEF), ce qui les mène à se comparer à des États tels que la Chine ou le Costa Rica sans pouvoir en tirer une analyse particulière.

La pérennité des échanges et la profondeur de la participation dépendent de l'échelle du territoire concerné : les initiatives nationales tendent à se limiter à une simple consultation, tandis que les échelles infranationales peuvent mettre en place une large participation et pérenniser le processus (Rondinella et al., 2017). Ainsi les indicateurs participatifs locaux apparaissent comme très pertinents car reflétant les territoires et les aspirations citoyennes, mais peu intéressants pour les décideurs aux échelles de gouvernance supérieures qui ne peuvent pas en tirer des conclusions directes plus générales, hors territoire original. À l'autre bout de la chaîne, les indicateurs internationaux apparaissent comme méthodologiquement robustes et offrent un fort potentiel en matière de communication, mais avec des variables trop générales pour adresser les problématiques locales. D'un côté, ce problème d'échelle permet de reconsidérer les processus de gouvernance et de dialoguer entre échelons décisionnels. De l'autre, les multiples initiatives à différentes échelles peuvent décourager certains décideurs à les mettre en place. En posant la question du rapport des indicateurs aux spécificités du territoire, qu'elles soient naturelles, sociales, économiques ou politiques, les enjeux scalaires révèlent la complexité du débat sur les indicateurs participatifs et soulèvent les problématiques de la représentativité de la mesure, du choix de sa méthodologie, des interactions entre les différents acteurs du débat et de la place de la société civile dans la sélection et la mise en œuvre des indicateurs alternatifs.

\section{Indicateurs et participation : un oxymore?}

Notre ambition a été de commencer une discussion commune entre les analystes de la participation et ceux travaillant sur les indicateurs, pour arriver à mieux comprendre si derrière l'engouement généralisé pour les indicateurs participatifs ne se cachaient pas finalement des sphères irréconciliables, transformant l'expression «indicateurs participatifs » en oxymore, autrement dit en contradiction ultime. Au-delà des promesses nombreuses et justifiées derrière la combinaison participation-indicateurs, le dossier a montré à quel point la prolifération des expériences participatives dans le domaine des indicateurs alternatifs n'est pas sans revêtir des aspects paradoxaux ou ambivalents.

[1] Torfaen Sustainability Index (https://www.torfaen.gov.uk/en/PestsPollutionHygiene/Sustainability/Sustainabledevelopment-general/Torfaen-Sustainability.aspx) et Caerphilly Sustainability Index (http://your.caerphilly.gov.uk/sustainablecaerphilly/sustainable-development/sustainable-council/measuring-progress), accès le 22/11/2017. 
À la lumière des contributions suivantes, on serait donc tenté de valider, du moins partiellement, l'hypothèse postulant que l'expression « indicateurs participatifs » relève d'un oxymore. En effet, les multiples approches qui ont vocation à démocratiser la mesure sont confrontées à de nombreux problèmes, dilemmes et paradoxes les empêchant d'atteindre leur but. Afin que les indicateurs participatifs parviennent dans un futur proche et dans la pratique à réconcilier ces paradoxes et défis - à savoir l'ouverture avec la fermeture, le consensus avec le dissensus, l'objectivité avec la subjectivité, le local avec le global, l'utilisation directe et l'apprentissage collectif -, le travail d'analyse croisée entre indicateurs alternatifs et processus participatifs mériterait de se focaliser sur un certain nombre de chantiers prioritaires.

\section{Préoccupations méthodologiques}

Le premier chantier concerne la clarification de la méthodologie à mettre en place lors de processus participatifs autour d'indicateurs. En effet, ce qui frappe à la lecture des contributions est la grande diversité d'approches proposées pour le même objectif, en termes de temporalité (quand faire intervenir la participation dans le cycle de vie de l'indicateur ?), de public cible (cherche-t-on un groupe restreint ou élargi, homogène ou hétérogène ?), de mode participatif (veut-on axer la participation sur la lexicométrie, le débat, le questionnaire ?), etc. Si les thèses des auteurs de ce dossier ne se rejoignent pas forcément à propos du public cible ou des modes participatifs, plusieurs contributions mettent l'accent sur l'importance d'intégrer la participation dans les différents « temps » de l'indicateur: lors de son élaboration bien sûr, mais aussi dans ses processus de suivi, de modification et d'utilisation. La littérature en la matière souligne l'importance de la participation au début du processus, par exemple lors des discussions portant sur le cadrage et la définition du développement durable (voir notamment Rosenström, Kyllönen, 2007). Comme le démontrent Le Roy et Ottaviani (dans ce dossier), la participation peut jouer une fonction importante mais différenciée selon l'étape du processus de la vie de l'indicateur. Tout en sachant que le contexte spécifique du cadre participatif est fondamental dans le succès ou l'échec de la démarche let les auteurs de ce numéro nous le rappellent), il conviendrait d'analyser de manière plus consolidée les démarches méthodologiques (et donc aussi procédurales) à adopter.

\section{Préoccupations épistémologiques}

Une observation évidente à la lumière des contributions du dossier concerne le pragmatisme ambiant qui régit les approches épistémologiques adoptées par les auteurs. Les textes de Jany-Catrice et Pinaud, de Le Roy et Ottaviani, ainsi que de Renault s'inscrivent tantôt pleinement tantôt implicitement dans une démarche pragmatiste. L'analyse future des indicateurs participatifs devrait plus fortement se préoccuper d’opérer des choix épistémologiques précis, en mobilisant 
par exemple des concepts et méthodologies de la sociologie pragmatique lvoir par exemple Chateauraynaud, Debaz, 2017). Une telle démarche permettrait d'aborder la question des influences sociétales issues des processus participatifs autour des indicateurs - comme le dit Renault, passer des «comptes » aux « contes»-, en étudiant l'articulation entre un ensemble de facteurs internes et externes à ces dispositifs participatifs. À l'instar du concept de « la portée de l'argumentation » (Chateauraynaud, 2011 ; Fourniau, Chateauraynaud, 2011), on pourrait ainsi mettre à l'épreuve entre autres la notion de "portée de l'indicateur » comme une résultante des articulations entre l'indicateur, ses porteurs et ses contextes. Par ailleurs, il persiste certainement une tradition unique et reconnue internationalement qui utilise des lentilles de la sociologie politique imprimée par des approches longitudinales, quasiment historiques, sur les objets de la mesure. Vanoli $(2002)$ et Desrosières $(1993,2008,2015)$ représentent une classe particulière d'administrateurs de la mesure len l'occurrence économiquel qui ont mené une vie pleine d'investigations et d'analyses profondes de la signification politique de la mesure. Aujourd'hui, le monde des chercheurs sur les indicateurs est tout aussi plein d'exemples de personnes, dont Jean Gadrey et le Forum pour d'autres indicateurs de richesse (FAIR), qui mènent aussi une vie d'activiste de la mesure. Les rôles des uns et des autres dans le domaine des indicateurs ont toujours eu tendance à être peu cimentés; un ancrage plus profond, plus épistémologique, de cette évidence pourrait aider à mieux structurer les indicateurs participatifs.

\section{Questionner les institutions de la mesure}

En troisième point, bien que les contributions de ce dossier donnent une large place aux réflexions concernant les rôles des experts économiques et des ingénieurs, elles abordent peu le rôle pourtant central des statisticiens en tant qu'acteurs majeurs de la configuration des mesures. De même, les articles de ce dossier sont principalement centrés sur les échelles de gouvernance infranationales, laissant de côté les défis considérables de la participation dans la production et l'usage des indicateurs au niveau international. Néanmoins, les bureaux de statistiques nationaux et internationaux (Insee en France, Eurostat en Europel sont des acteurs-clés dans le formatage et la structuration des processus de production des indicateurs, et sont sous une pression croissante de la part des mondes politique, associatif et scientifique pour renforcer l'engagement des citoyens dans la production des indicateurs. Il s'agit là d'un défi de taille pour ces acteurs, dont l'autorité et l'identité propre dépendent de leur capacité à produire des statistiques et indicateurs fiables et stables à travers le temps. Ce défi concerne non seulement l'interaction et le partage des tâches entre experts et citoyens, mais aussi le traitement de données de qualité aléatoire. Par exemple, pour faciliter la participation et l'inclusion d'une diversité des types de savoirs, faudrait-il assouplir les normes conventionnelles et hautement contraignantes qui gouvernent la production des indicateurs et statistiques officiels ? Baisser le seuil de rigueur exigé ou encore réagir plus rapidement à des impulsions régies 
par les contextes politiques et sociétaux risquerait - en politisant la configuration des mesures - de diminuer la confiance envers les indicateurs produits, tandis que le maintien de critères stricts irait à l'encontre des objectifs de légitimité auprès de publics variés. Pour l'instant, le conservatisme des offices statistiques tout comme les pressions budgétaires sensibles constituent des freins à l'innovation nécessaire pour le développement de procédures plus participatives (Van Tuinen, 2009 ; Chancel et al., 2014). Ainsi positionner ces acteurs au centre des efforts pour renforcer les processus participatifs de conception et d'utilisation des indicateurs apparaît comme incontournable.

\section{Conclusion}

Les Indicateurs de Développement Durable (IDD), les Nouveaux Indicateurs de Richesse (NIR) et les Indicateurs de Bien-Être (IBE) figurent parmi les efforts les plus influents ayant pour vocation d'utiliser des dispositifs de gouvernance classiques afin de changer nos trajectoires sociétales de manière radicale vers plus de durabilité, d'égalité, de partage, de respect. Souvent l'impératif d'associer de la participation à ces processus de configuration et d'utilisation d'indicateurs est une des garanties avancées pour ne pas tomber dans le jeu des acteurs dominants, et pour combler le décalage entre les logiques gestionnaires et les promesses émancipatrices des indicateurs. En théorie, tous les acteurs sont, du moins dans le discours, favorables à la production d'indicateurs participatifs ne serait-ce que pour poursuivre les objectifs les plus simples, à savoir pour intégrer des savoirs profanes dans la définition des objets sous mesure et pour renforcer leur utilisation.

Séparément, participation et indicateurs ont fait couler beaucoup d'encre, avec pour chaque objet ses atouts et ses faiblesses. Mais que peut donner l'association de ces deux concepts multiformes? Les indicateurs présentent des défis particuliers pour les processus participatifs; et inversement, les processus participatifs peuvent impacter la forme, le bien-fondé, l'utilisation et l'impact de l'indicateur. Peu de travaux abordant spécifiquement cette interaction, nous avons voulu ici explorer les problématiques, les enjeux et les difficultés que pose la question des indicateurs participatifs. D'un côté, l'indicateur représenterait la mesure, la réduction de la complexité, la fermeture, le consensus; de l'autre, la participation viserait l'ouverture, le dialogue, l'hétérogénéité, la diversité. Afin de savoir si l'expression « indicateurs participatifs » représente ou non un oxymore, nous avons voulu voir au-delà de l'engouement généralisé pour cette pratique et discuter empiriquement les vertus supposées des indicateurs participatifs, avec l'appui des contributions suivantes embrassant une variété d'expérimentations sur le sujet.

Ce dossier permet au lecteur de se faire une idée précise de certaines des plus ambitieuses initiatives (francophones, pour l'essentiel) de ces dernières années. Les analyses proposées dans ce dossier, liées à une analyse de la littérature 
plus générale, ont fait émerger six promesses qui cadrent les contradictions et les opportunités que recèlent les indicateurs participatifs. Le dossier montre en particulier que les indicateurs participatifs sont source de confrontations entre expertises institutionnelles, académiques et citoyennes (1); entre objectivité et subjectivité, ainsi qu'entre données quantitatives et qualitatives (2); entre impératifs d'ouverture et d'apprentissage qui s'opposent aux besoins de fermeture et de cadrage (3). Le dossier montre également que de ces trois types de confrontations découlent les résultats tangibles, positifs ou négatifs, de la mise en participation des indicateurs alternatifs. En effet, les processus participatifs autour des indicateurs peuvent accentuer, ou atténuer, les asymétries de pouvoir (4); limiter l'utilisation des indicateurs dans la prise de décision politique ou fonder de nouveaux registres de gouvernance réflexive (5); et conduire à une dissociation scalaire des enjeux ou au contraire à l'émergence d'une intégration améliorée des échelles territoriales (6). Au final, le défi de taille des indicateurs participatifs consiste à faire coexister pertinence statistique et recherche de sens, instrument de mesure et complexité sociale.

Terminons cette réflexion introductive au dossier sur les promesses et dilemmes associés aux indicateurs participatifs par une évidence que le lecteur trouvera dans l'ensemble des contributions : les auteurs, quoique conscients des difficultés, restent optimistes sur l'intérêt de mettre en place de la participation dans la définition de la mesure. Les indicateurs alternatifs et participatifs renferment une dimension politique à un degré tel qu'ils en deviennent des objets politiques, ce qui fait que la multitude des expérimentations dans le domaine reste encourageante et très positive, car pourvoyeuse de nombreux effets rebonds, tels les apprentissages collectifs ou les interrogations sur les valeurs sociétales. Comme le constatent Jany-Catrice et Pinaud, si les indicateurs nouveaux ne garantissent pas en tous lieux leur usage direct par le monde politique, ils offrent néanmoins une prise de recul critique sur les politiques en place dont peut se ressaisir la société civile. La promesse de la démocratisation se meut donc en opportunité de la contestation politique. Par ailleurs, en insérant une forme d'incertitude sur ce qu'il faut mesurer, la participation incite à une remise en cause régulière de l'automaticité des démarches de suivi par le chiffre. Si ce mécanisme pose un problème évident aux institutions de la mesure qui tendent à protéger les indicateurs d'une trop volatile contextualisation, et des critiques de leur politisation, Vergote et Petit rappellent qu'un indicateur doit rester un support de réflexion et ne doit pas devenir une finalité. Non figé, il permet d'étudier toute une diversité d'arrangements sociotechniques. Ainsi la participation autour des indicateurs peut-elle être très profitable pour la société dans son ensemble si elle est organisée au service d'un questionnement sur les finalités de l'action collective, afin de guider celle-ci vers la controverse citoyenne. 


\section{Bibliographie}

Argyris C., Schön D., 1978, Organisational learning : A theory of action perspective, Reading (MA), Addison Wesley.

Barthe Y., 2002, «Les conséquences inattendues des procédures de concertation. Retour d'expérience dans le domaine des déchets nucléaires », Séminaire Concertation, décision, environnement, Quels cadres d'évaluation des dispositifs de concertation?, séance n 8, mercredi 26 juin 2002.

Batterbury S., Forsyth T., 1997, « Environmental transformations in developing countries: hybrid research and democratic policy », Geographical journal, 163 (2), p. 126-132.

Bell S., Morse S., 2003, Measuring sustainability. Learning from doing, Londres, Earthscan.

Bell S., Morse S., 2008, Sustainability Indicators : Measuring the Immeasurable?, Londres, Earthscan.

Bell S., Morse S., 2011, «An analysis of the factors influencing the use of indicators in the European Union », Local Environment: The International Journal of Justice and Sustainability, 16 (3), p. 281-302.

Bogner A., 2012, "The Paradox of Participation Experiments », Science, Technology, \& Human Values, 37 (5), p. 506-527.

Bora A., Hausendorf H., 2006, «Participatory Science Governance Revisited: Normative Expectations versus Empirical Evidence», Science and Public Policy, 33 (7), p. 478-488.

Bradshaw B., 2003, «Questioning the credibility and capacity of communitybased resource management », The Canadian Geographer, 47 (2), p. 137-150.

Bruno I., Didier E., Prévieux J., 2014, Statactivisme. Comment lutter avec des nombres, Paris, La Découverte.

Bruno I., Jany-Catrice F., Touchelay B. (dir.), 2016, The Social Sciences of Quantification. From Politics of Large Numbers to Target-Driven Policies, Londres, Springer.

Chancel L., Thiry G., Demailly D., 2014, « Les nouveaux indicateurs de prospérité : pour quoi faire? Enseignements de six expériences nationales », Study, $n^{\circ} 04 / 14$, septembre 2014, 32 p.

Chateauraynaud F., 2011, Argumenter dans un champ de forces. Essai de balistique sociologique, Paris, Éditions Pétra.

Chateauraynaud F., Debaz J., 2017, Aux bords de l'irréversible. Sociologie pragmatique des transformations, Paris, Éditions Pétra.

Citton Y., 2007, Lire, interpréter, actualiser. Pourquoi les études littéraires ?, Paris, Éditions Amsterdam.

Cleaver F., 1999, «Paradoxes of participation: questioning participatory approaches to development », Journal of International Development, 11 (4), p. 597-612. 
Conrad C. C., Hilchey K. G., 2011, «A review of citizen science and communitybased environmental monitoring : issues and opportunities », Environmental Monitoring and Assessment, 176 (1), p. 273-291.

Cooke B., Kothari U. (dir.), 2001, Participation: The New Tyranny?, Londres, Zed Books.

Desrosières A., 1993, La politique des grands nombres. Histoire de la raison statistique. Paris, La Découverte.

Desrosières A., 2008, L'argument statistique, Paris, Presses des Mines.

Desrosières A., 2015, «Retroaction : how indicators feed back onto quantified actors », in R. Rottenburg, S. E. Merry, S.-J. Park, J. Mugler (dir.), The World of indicators: The making of Governmental Knowledge through Quantification, Cambridge/New York, Cambridge University Press.

Fourniau J.-M., Chateauraynaud F., 2011, « La portée de la participation dans une approche de balistique sociologique. », Billet publié sur le site Portée de la concertation : Sociologie des effets de la participation du public aux processus décisionnels, 8 janvier 2011, https://concertation.hypotheses.org/277 laccès le 15/10/2017).

Fraser E., Dougill A., Mabee W., Reed M., McAlpine P., 2006, « Bottom up and top down: analysis of participatory processes for sustainability indicator identification as a pathway to community empowerment and sustainable environmental management », Journal of Environmental Management, 78 (2), p. 114-127.

Gadrey J., Jany-Catrice F., 2012, Les nouveaux indicateurs de richesse, 3e éd., Paris, La Découverte.

Godin B., 2002, «Outline for a History of Science Measurement », Science, Technology and Human Values, 27 (1), p. 3-27.

Gouveia C., Fonseca A., Camara A., Ferreira F., 2004, «Promoting the use of environmental data collected by concerned citizens through information and communication technologies », Journal of Environmental Management, 71 (2), p. 135-154.

Hall J., Rickard L., 2013, People, progress and participation. How initiatives measuring social progress yields benefits beyond better metrics, Gütersloh, Bertelsmann Stiftung.

Hardi P., Zdan T., 1997, Assessing sustainable development. Principles in practice, Winnipeg (Canada), The International Institute for Sustainable Development.

Hendriks C. M., 2006, «Integrated Deliberation : Reconciling Civil Society's Dual Role in Deliberative Democracy », Political Studies, 54 (3), p. 486-508.

Hezri A. A., 2006, Connecting Sustainability Indicators To Policy Systems, thèse pour le doctorat en sciences philosophiques, The Australian National University.

Hezri A. A., Dovers S. R., 2006, « Sustainability indicators, policy and governance : Issues for ecological economics », Ecological Economics, 60 (1), p. 86-99.

Hezri A.A., 2004, «Sustainability indicators system and policy processes in Malaysia : a framework for utilisation and learning », Journal of Environmental Management, 73 (4), p. 357-371. 
Hezri A. A., Hasan M. N., 2004, « Management framework for sustainable development indicators in the State of Selangor, Malaysia », Ecological Indicators, 4 (4), p. 287-304.

Hisschemöller M., Cuppen E., 2015, «Participatory Assessment: Tools for Empowering, Learning and Legitimating?», in A. Jordan, J.R. Turnpenny (dir.), The Tools of Policy Formulation: Actors, Capacities, Venues and Effects, Cheltenham, Edward Elgar, p. 33-51.

Holden M., 2013, « Sustainability indicator systems within urban governance: Usability analysis of sustainability indicator systems as boundary objects », Ecological Indicators, 32, p. 89-96.

Hood C., 2007, «Public service management by numbers: Why does it vary? Where has it come from ? What are the gaps and the puzzles? », Public Money \& Management, 27 (2), p. 95-102.

Innes J. E., Booher D. E., 2000, « Indicators for sustainable communities : A strategy building on complexity theory and distributed intelligence », Planning Theory \& Practice, 1 (2), p. 173-186.

IWEPS, 2015, Indicateurs complémentaires au PIB. L'indice des conditions de bien-être en Wallonie-ICBE, $2^{e}$ exercice, juin 2015, https://www.iweps.be/wpcontent/uploads/2017/01/ICBE_2015_rapport_de_recherche_final-19aout. pdf laccès le 10/09/2016).

Jackson P. M., 2011, « Governance by numbers : what have we learned over the past 30 years ? », Public Money \& Management, 31 (1), p. 13-26.

Jany-Catrice F. (dir.), 2007 [2009], «Inégalités et pauvreté dans la région NordPas de Calais. Baromètres », Étude prospective régionale, n²12, Rapport pour le Conseil régional Nord-Pas de Calais.

Jany-Catrice F., 2009, « The French Regions and Their Social Health », Social indicators research, 93 (2), p. 377-391.

Jany-Catrice F., 2013, « Indicateurs de richesse », in N. Postel, R. Sobel (dir.), Dictionnaire critique de la RSE, Villeneuve-d'Ascq. Presses Universitaires du Septentrion.

Lascoumes P., Le Galès P. (dir.), 2005, Gouverner par les instruments, Paris, Presses de Sciences Po.

Le Play F., 1878, La réforme sociale en France déduite de l'observation comparée des peuples européens, Tours, Alfred Mame et fils.

Lehtonen M., 2013, «The non-use and influence of UK Energy Sector Indicators », Ecological Indicators, 35, p. 24-34.

Lehtonen M., Sébastien L., Bauler T., 2016, « The multiple roles of sustainability indicators in informational governance : between intended use and unanticipated influence », Current Opinion in Environmental Sustainability, 18, p. 1-9.

Levidow L., 2007, European Public Participation as Risk Governance : Enhancing Democratic Accountability for Agbiotech Policy?, East Asian Science, Technology and Society : An International Journal, 1 (1), p. 19-51. 
Lyytimäki J., Tapio P., Varho V., Söderman T., 2013, « The use, non-use and misuse of indicators in sustainability assessment and communication », International Journal of Sustainable Development \& World Ecology, 20 (5), p. 385-393.

Magee L., Scerri A., James P., Thom J. A., Padgham L., Hickmott S., Deng H., Cahill F., 2013, « Reframing social sustainability reporting : towards an engaged approach », Environment, Development and Sustainability, 15 (1), p. 225-243.

Mansbridge J., 1999, « Everyday Talk in the Deliberative System », in S. Macedo (dir.), Deliberative Politics: Essays on Democracy and Disagreement, Oxford, Oxford University Press, p. 211-239.

Martin B. R., 2016, «Twenty challenges for innovation studies», Science and Public Policy, 43 (3), p. 432-450.

Mascarenhas A, Nunes L. M., Ramos T. B., 2014, « Exploring the self-assessment of sustainability indicators by different stakeholders », Ecological Indicators, 39, p. 75-83.

Mazeaud A., Sa Vilas Boas M.-H., Berthomé G.-E.-K., 2012, « Penser les effets de la participation sur l'action publique à partir de ses impensés », Participations, 2, p. 5-29.

McCool S. F., Stankey G. H., 2004, «Indicators of Sustainability: Challenges and Opportunities at the Interface of Science and Policy», Environmental Management, 33 (3), p. 294-305.

Méda D., 1999, Qu'est-ce que la richesse?, Paris, Aubier.

Méda D., Jany-Catrice F., 2013, «Les nouvelles mesures des performances économiques et du progrès social. Le risque de l'économicisme », Revue $d u$ MAUSS, 41 (1), p. 371-397.

Mickwitz P., Melanen M., 2009, «The role of co-operation between academia and policymakers for the development and use of sustainability indicators - a case from the Finnish Kymenlaakso Region », Journal of Cleaner Production, 17 (12), p. 1086-1100.

Mitroff I. I., Emshoff J. R., 1979, « On strategic assumption-making : A dialectical approach to policy and planning », The Academy of Management Review, 4 (1), p. 1-12.

Mouffe C., 1999, «Deliberative Democracy or Agonistic Pluralism ?», Social Research, 66 (3), p. 745-758.

Nygren A., 1999, « Local Knowledge in the Environment-Development Discourse. From dichotomies to situated knowledges », Critique of anthropology, 19 (3), p. 267-288.

Ortega-Cerdá M., 2005, «Sustainability Indicators as Discursive Elements », 6th International Conference of the European Society for Ecological Economics, Lisbonne, 14-17 juin 2005.

Parkinson J., 2006, Deliberating in the real world : problems of legitimacy in deliberative democracy, Oxford (NY), Oxford University Press.

Parris T. M., Kates R. W., 2003, «Characterizing and Measuring Sustainable Development », Annual Review of Environmental Resources, 28, p. 1-28. 
Petersen A. C., Cath A., Hage M., Kunseler E., Van der Sluijs J. P., 2011, « PostNormal Science in Practice at the Netherlands Environmental Assessment Agency », Science, Technology \& Human Values, 36 (3), p. 362-388.

Pintér L., Hardi P., Bartelmus P., 2005, Indicators of Sustainable Development: Proposals for a Way Forward prepared for the United Nations Division for Sustainable Development (UN-DSD), Winnipeg, International Institute for Sustainable Development (IISD).

POINT, 2011, Policy Influence of Indicators : Scientific Summary Report, EU FP7 Grant Agreement number 217207, http://cordis.europa.eu/docs/results/217207/ final1-point-final-report.pdf laccès le 10/11/2017).

Rafols I., Ciarli T., Van Zwanenberg P., Stirling A., 2012, « Towards Indicators for “Opening Up” Science and Technology Policy », Internet, Politics, Policy 2012 : Big Data, Big Challenges?. University of Oxford, Oxford Internet Institute, 20-21 septembre 2012.

Rauschmayer F., Wittmer H., 2006, «Evaluating deliberative and analytical methods for the resolution of environmental conflicts », Land Use Policy, 23 (1), p. 108-122.

Reed M. S., Fraser E. D. G., Dougill A. J., 2006, « An adaptive learning process for developing and applying sustainability indicators with local communities », Ecological Economics, 59 (4), p. 406-418.

Rondinella T., Segre E., Zola D., 2017, «Participative Processes for Measuring Progress: Deliberation, Consultation and the Role of Civil Society », Social Indicators Research, 130 (3), p. 959-982.

Rosenström U., Kyllönen S., 2007, « Impacts of a participatory approach to developing national level sustainable development indicators in Finland », Journal of Environmental Management, 84 (3), p. 282-298.

Rottenburg R., Merry S. E., 2015, « A world of indicators : the making of governmental knowledge through quantification », in R. Rottenburg, S.E. Merry, S.-J. Park, J. Mugler (dir.), The World of Indicators : The Making of Governmental Knowledge through Quantification, Cambridge, Cambridge University Press.

Russell S. L., Thomson I., 2009, « Analysing the role of sustainable development indicators in accounting for and constructing a Sustainable Scotland », Accounting Forum, 33 (3), p. 225-244.

Ruyters C., Laffut M., Defays D., Colicis 0., 2011, «Élaboration concertée d'indicateurs de bien-être dans les communes wallonnes. Partie 1 : la genèse du projet et les premiers résultats de l'expérience en cours », Working Papers de l'IWEPS, 3, septembre 2011.

Rydin Y., 2007b, «Indicators as a governmental technology? The lessons of community-based sustainability indicator projects », Environment and Planning D : Society and Space, 25 (4), p. 610-624.

Rydin Y., Holman N., Wolff E., 2003, « Local sustainability indicators », Local Environment, 8 (6), p. 581-589.

Rydin, Y., 2007a, « Re-Examining the Role of Knowledge Within Planning Theory », Planning Theory, 6 (1), p. 52-68. 
Salvaris M., 2000, Community and social indicators. How citizens can measure progress (An overview of social and community indicator projects in Australia and internationally), Australie, Institute for Social Research, http://library.bsl.org. au/jspui/bitstream/123456789/362/1/Community\%20Social\%20Indicators. pdf laccès le 08/11/2017).

Seaford C., 2013, «The multiple uses of subjective well-being indicators », Social Indicator Research, 114 (1), p. 29-43.

Sébastien L., Bauler T., 2013, «Use and influence of composite indicators for sustainable development at the EU-level », Ecological Indicators, 35, p. 3-12.

Sébastien L., Lehtonen M., Bauler T., 2014, «Can indicators bridge the gap between science and policy? An exploration of the (non) use and (non) influence of indicators in EU and UK policymaking », Nature \& Culture, 9 (3), p. 316-343.

Söderman T., Kopperoinen L., Yli-Pelkonen V., Shemeikka P., 2012, « Ecosystem services criteria for sustainable development in urban regions », Journal of Environmental Assessment Policy and Management, 14 (2), p. 1-48.

Star S. L., 2010, «This is Not a Boundary Object : Reflections on the Origin of a Concept », Science, Technology, \& Human Values, 35 (5), p. 601-617.

Stiglitz J. E., Sen A., Fitoussi J.-P., 2010, Mismeasuring our lives. Why GDP doesn't add up. The Report by the Commission on the Measurement of Economic Performance and Social Progress, New York/Londres, New Press.

Stirling A., 2008, " "Opening Up" and "Closing Down": Power, Participation, and Pluralism in the Social Appraisal of Technology », Science, Technology, \& Human Values, 33 (2), p. 262-294.

Theys J., 2002, «L'approche territoriale du "développement durable", condition d'une prise en compte de sa dimension sociale », Développement durable et territoires, http://developpementdurable.revues.org/1475 (accès le 09/11/2017).

Thiry G., 2012, Au-delà du PIB : un tournant historique. Enjeux méthodologiques, théoriques et épistémologiques de la quantification, thèse pour le doctorat en sciences économiques, Université catholique de Louvain.

Thiry G., Sébastien L., Bauler T., 2016, « Ce que révèlent les discours des acteurs institutionnels sur un "au-delà du PIB" », Natures Sciences Sociétés, 24 (1), p. 3-14.

Tournay V., 2015, «Faut-il restituer la complexité du monde observé pour le comprendre? Apports et limites d'une anthropologie de l'évaluation», SociologieS, http://sociologies.revues.org/4895 (accès le 09/11/2017).

Turcu C., 2013, « Re-thinking sustainability indicators : local perspectives of urban sustainability », Journal of Environmental Planning and Management, 56 (5), p. 695-719.

Turnhout E., 2009, « The effectiveness of boundary objects : the case of ecological indicators », Science and Public Policy, 36 (5), p. 403-412.

Valentin A., Spangenberg, J. H., 2000, « A guide to community sustainability indicators », Environmental Impact Assessment Review, 20 (3), p. 381-392. 
Van den Hove S., 2006, «Between consensus and compromise : acknowledging the negotiation dimension in participatory approaches », Land Use Policy, 23 (1), p. 10-17.

Van Tuinen H. K., 2009, «Innovative Statistics to Improve Our Notion of Reality », Journal of Official Statistics, 25 (4), p. 431-465.

Vanoli A., 2002, Une histoire de la comptabilité nationale, Paris, La Découverte.

Venetoulis J., Cobb C., 1998, The Genuine Progress Indicator 1950-2002 2004 Update), San Francisco, Redefining Progress, http://rprogress.org/publications/2004/gpi_march2004update.pdf laccès le 15/10/2017).

Voss J. P., Bauknecht D., Kemp R. (dir.), 2006, Reflexive Governance for Sustainable Development, Cheltenham (UK)/Northampton (MA), Edward Elgar.

Voss J. P., Freeman R., 2016, «Introduction : Knowing Governance », in J. P. Voss, R. Freeman (dir.), 2016, Knowing Governance: The Epistemic Construction of Political Order, Basingstoke, Palgrave Macmillan.

Zittoun P., 2006, « Indicateurs et cartographie dynamique du bruit, un instrument de reconfiguration des politiques publiques ? », Développement durable et territoires, dossier 8 , http://developpementdurable.revues.org/index3261.html laccès le 07/11/2017).

Léa Sébastien est maître de conférences en géographie à l'Université Toulouse II et chercheure au laboratoire CNRS GEODE depuis 2010. Ses trois thèmes majeurs de recherche sont l'usage politique des indicateurs de développement durable, les résistances aux projets d'aménagement et les implications de l'attachement au lieu. Après des études effectuées à l'Université McGill au Canada, elle a réalisé une thèse à l'École des Mines en 2006 au cours de laquelle elle mit sur pied un modèle d'aide à la négociation environnementale intitulé l'Acteur en 4 Dimensions. Léa Sébastien a effectué un postdoctorat en 2007 à l'Ifremer sur les jeux d'acteurs en zones humides, puis été chercheure senior à l'Université de Bruxelles de 2008 à 2010 où elle était en charge de projets européens FP7 (POINT, CEECEC, BRAINPOOL). Depuis la publication d'un ouvrage scientifique en 2002 portant sur les potentialités de gestion durable en forêt privée (Forêt cherche propriétaire pour relation durable, Éditions ECOFOR), Léa Sébastien a publié plus d'une vingtaine d'articles en français et en anglais sur les problématiques émergentes des indicateurs de durabilité, des conflits environnementaux et de l'attachement au lieu.

Markku Lehtonen est docteur en économie de l'environnement de l'Université de Versailles Saint-Quentin-en-Yvelines (2005) et titulaire d'un master en gestion de l'environnement (Université de Helsinki, 1994). Entre 2005 et 2012, il a travaillé en tant que Research Fellow au SPRU (Science Policy Research Unit) de l'Université du Sussex, de 2012 à 2014 à l'Ifris (Institut francilien recherche sciences sociétés), et depuis 2009 comme chercheur associé dans le Groupe de sociologie pragmatique et réflexive (GSPR) de l'EHESS. Ses domaines d'expertise incluent 
la démocratie délibérative et participative dans les politiques environnementales, énergétiques et d'aménagement, les controverses publiques, l'évaluation des «mégaprojets » et le rôle de l'expertise (évaluations, indicateurs) dans la gouvernance.

Tom Bauler est professeur d'économie écologique à l'Université libre de Bruxelles (ULB) et chercheur au sein du Centre d'études du développement durable du Département de géosciences, environnement et sociétés de la Faculté des sciences. Après une formation initiale en tant qu'économiste (Université Louis Pasteur, Strasbourg, France), suivie par l'étude des interactions entre science et société dans le domaine de la politique environnementale (MA Sciences, techniques et sociétés, École polytechnique fédérale de Lausanne, Suisse), ses intérêts pour les questions environnementales se sont transformés en une thèse de doctorat à l'ULB sur les questions de l'utilisation des indicateurs de développement durable dans les processus de prise de décision institutionnelle. Il s'intéresse aux liens entre la politique environnementale et l'économie. Partant d'une tradition d'économie politique, et d'une base disciplinaire en économie écologique (Ecological Economics) en particulier, ses recherches associent des approches de la sociologie politique et de la sociologie des sciences et des techniques (STS). Ses principaux champs de recherche sont l'institutionnalisation, l'évaluation et les usages des instruments d'action publique dans le cadre de la politique environnementale, ainsi que la gouvernance, la traduction et la capture de l'innovation sociale et sociotechnique par les acteurs sociétaux et politiques. 